\title{
POSTERIOR REVERSIBLE ENCEPHALOPATHY SYNDROME (PRES) \\ A case with bilateral loss of vision during an elective LSCS
}

\section{F. Maudarbaccus ${ }^{1}$, C. Chiscaru ${ }^{1}$, A. Woolhead Conf. O. P. Teodorescu²}

\section{Case report}

A 42-year old lady, NKDA, G3P2, 39/40, presented for an elective LSCS (IUGR, oligohydramnios,). Three weeks prior to presentation, she had been observed for borderline hypertension without proteinuria. She underwent an uneventful spinal anaesthetic, but soon after, required phenylephrine boli for hypotensive episodes. She experienced an exaggerated response to standard dose phenylephrine and her BP reached $215 / 110$ from a low 70/40. A healthy but small for dates baby was delivered, but soon after, the mother complained of severe headaches with total loss of bilateral vision.

\section{What is PRES?}

Posterior reversible encephalopathy syndrome (PRES) is a constellation of neurological symptoms and radiological abnormalities consisting of headaches, altered mental status, visual disturbances/blindness and seizures. ${ }^{[1,2]}$ It is often associated with an abrupt increase in BP and has been described in a number of medical conditions, including hypertensive encephalopathy, preeclampsia/eclampsia, ARF/CRF, electrolyte disturbances and the use of cytotoxic and immunosuppressant drugs.

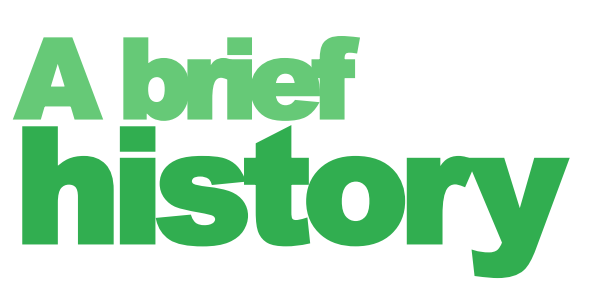

Obstetric

$\rightarrow \mathrm{G} 3 \mathrm{P} 2$

- Previous epidural analgesia X2 with uneventful SVD

- Postpartum depression

Medical

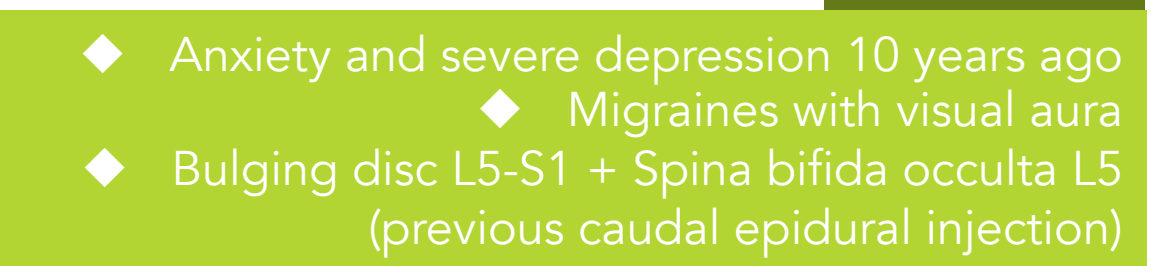

Medications

- Gaviscon

- Paracetamol

\section{Brain scans}

CT Brain performed 2 hours postop was inconclusive and warranted an MRI, which when performed 5 hours postop showed areas of hyper-intense signals in both occipital lobes and on right side of cerebellum extending out to cerebellar cortex on both diffusion and FLAIR sequence.

Repeat MRI 2 weeks after neurological insult showed complete disappearance of previously hyper-intense signals.
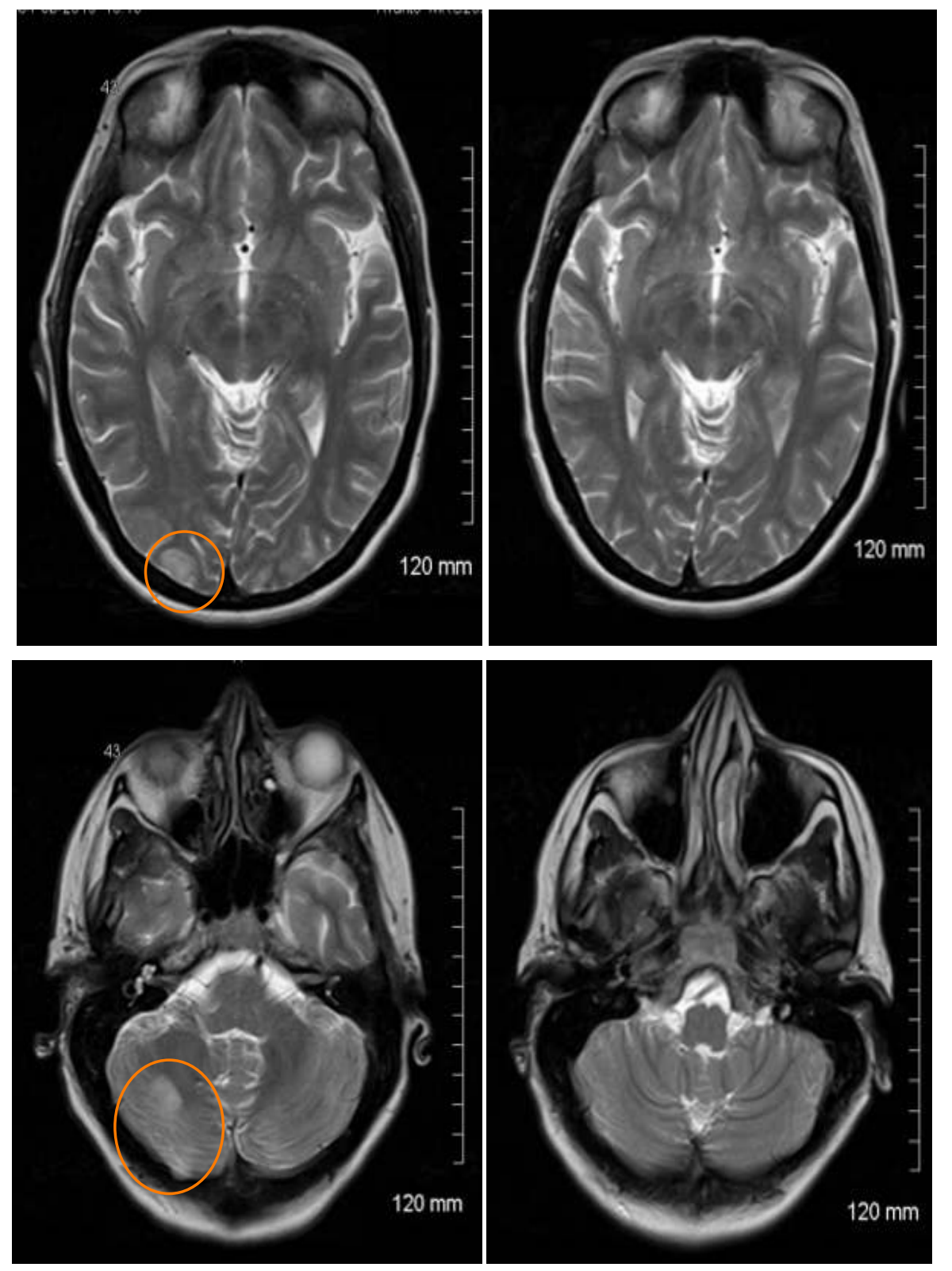

\section{Pathogenesis}

The pathophysiology of PRES is still very much poorly understood but seem to be related to a disordered cerebral autoregulation and endothelial dysfunction. The combination of acute hypertension and endothelial damage can lead to vasogenic oedema which is caused by the leakage of serum through capillary walls into the brain interstitium.

Interestingly, she did suffer from migraines with visual aura, as per her history. There seems to be a common pathophysiology for these disorders indicating that migraine aura can occur after
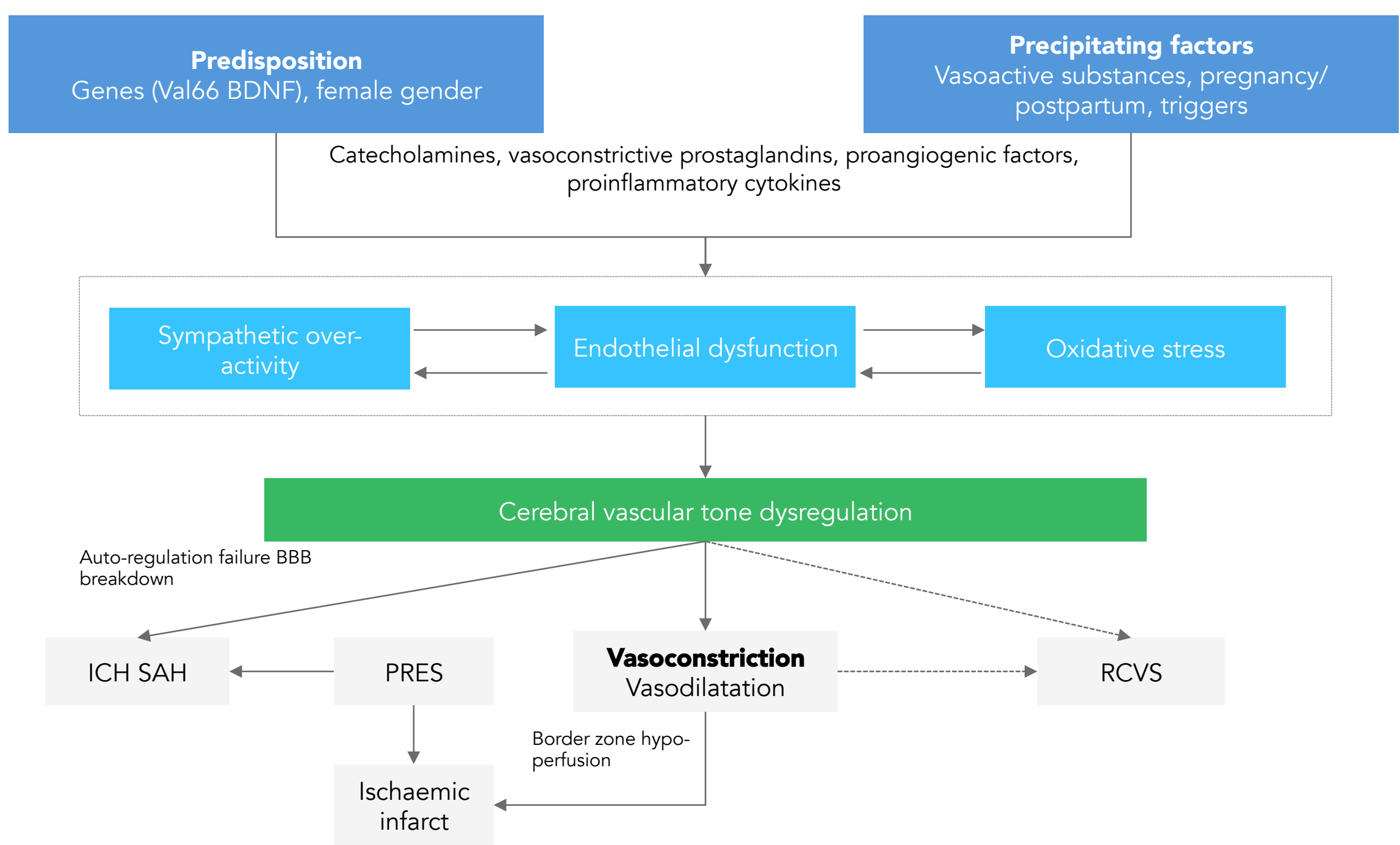

periods of hypoperfusion, due to local endothelial or smooth muscle dysfunction. ${ }^{[4]}$ However causative relationship between migraine with aura and cerebrovascular disorders is difficult to prove.

\section{Discussion \& management}

As per the revised criteria by the ACOG in 2013, preeclampsia refers to the new onset of hypertension and either proteinuria or endorgan dysfunction after 20 weeks of gestation in a previously normotensive woman.[3] Although our patients had no criteria for preeclampsia, her clinical course was highly suggestive of preeclampsia. She did have borderline hypertension but was not on any medications and had no proteinuria.

Initial investigations (FBC/Coags/U\&E) including workup for PET were all within normal ranges both preop and postop. Her fundi were normal on ophthalmoscopy and she had no other neurological deficit. Cranial nerve exam was also normal.

Upon institution of labetalol/magnesium infusion, she started to regain her vision slowly and the headaches started to decrease in intensity. Clinical improvement with complete resolution of symptoms was observed within 7 hours.

\section{Conctusion}

This case demonstrates that awareness of PRES is important and also highlights the potential reversibility of this condition. Cerebral MRI is the key investigation for diagnosis of PRES. Rapid recognition and institution of treatment is essential to avoid complications, which can
lead to permanent disability or even death.

\section{Precipitating factors} postpartum, triggers 